\title{
Observation of Moiré-like Fringes in HAADF-STEM Images of Heterostructures of Two-dimensional Materials
}

\author{
Danielle Reifsnyder Hickey ${ }^{1}$ and K. Andre Mkhoyan ${ }^{1}$ \\ ${ }^{1}$ Department of Chemical Engineering and Materials Science, University of Minnesota, Minneapolis, \\ MN, United States
}

Moiré patterns occur in conventional transmission electron microscopy (CTEM) and bright-field scanning transmission electron microscopy (BF-STEM) images due to the interference resulting from two sets of periodic features [1]. These Moiré fringes can provide useful insights into a number of materials' properties, such as the relative orientation of crystal lattices, the existence of strain at interfaces, and the presence of defects such as dislocations.

Traditionally, Moiré patterns are not observed in high-angle annular dark-field (HAADF-STEM) imaging. However, we report here the systematic observation of several examples of large-period features in aberration-corrected HAADF-STEM images of heterostructures of two-dimensional materials. We will discuss the origin of these features and the microscope conditions under which they have been observed. Our observations are counterintuitive at first glance, but they provide an interesting case study for unusual effects in STEM imaging.

The samples studied were $(\mathrm{Bi}, \mathrm{Sb})_{2} \mathrm{Te}_{3}$ films grown on hexagonal boron nitride $(\mathrm{h}-\mathrm{BN})$ by molecular beam epitaxy (MBE). These films exhibit grain growth with various orientations of the grains relative to the h-BN substrate, and they contain features such as dislocations and strain. Figure 1 shows a comparison of a $(\mathrm{Bi}, \mathrm{Sb})_{2} \mathrm{Te}_{3} / \mathrm{h}-\mathrm{BN}$ heterostructure imaged in CTEM and HAADF-STEM. This study was performed on an FEI Titan G2 60-300 S/TEM equipped with a Schottky X-FEG gun, operated at $200 \mathrm{kV}$, and an FEI Tecnai G2 F30, operated at $300 \mathrm{kV}$ [2].

\section{References:}

[1] DB Williams and CB Carter. "Transmission Electron Microscopy: A Textbook for Materials Science ( $2^{\text {nd }}$ Edition)", (Springer, New York, 2009).

[2] The authors gratefully acknowledge Dr. Joon Sue Lee and Prof. Nitin Samarth for providing the samples studied, as well as funding provided by C-SPIN, one of six centers of STARnet, a Semiconductor Research Corporation program, sponsored by MARCO and DARPA. 

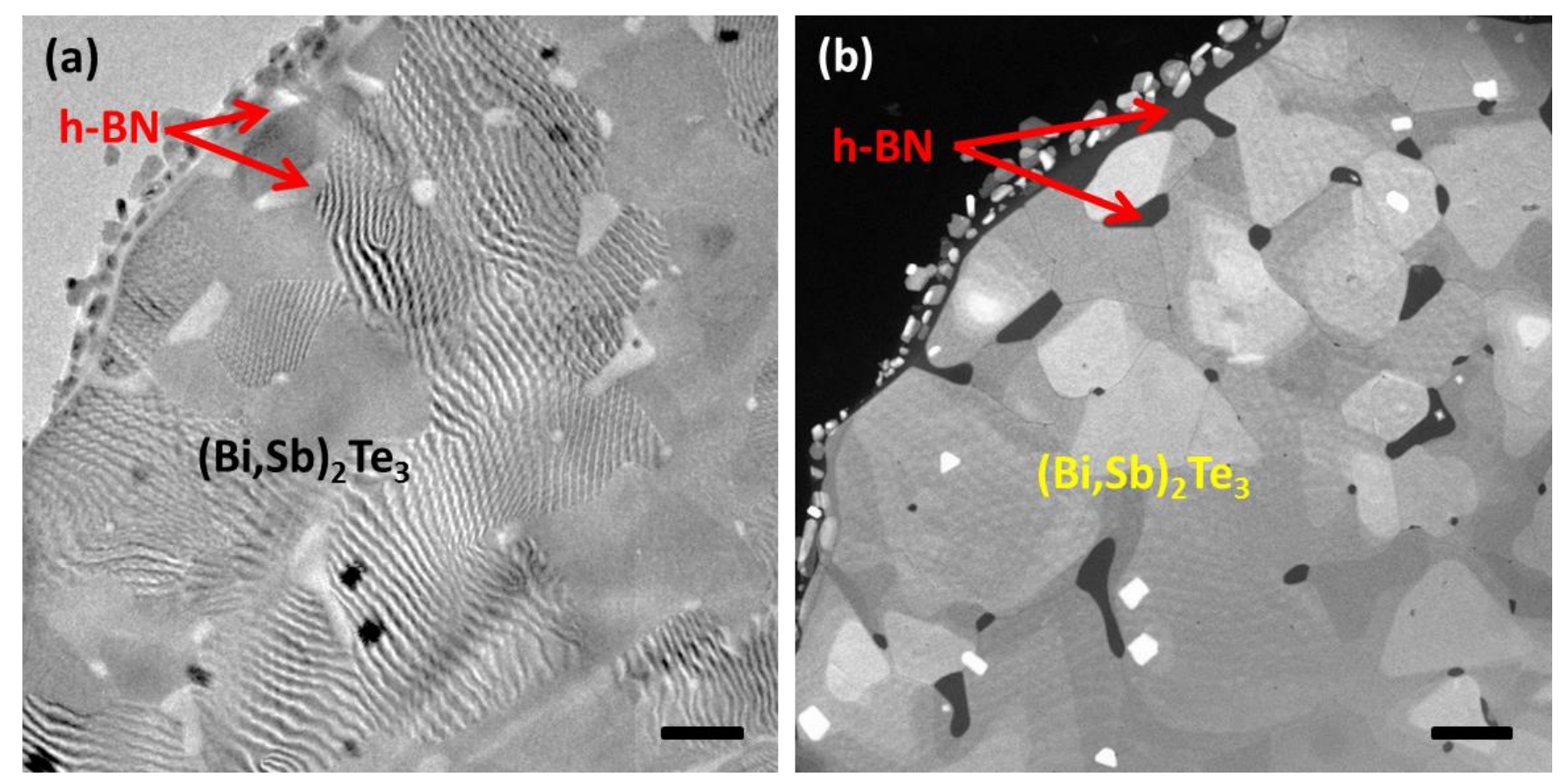

Figure 1. Images of a $(\mathrm{Bi}, \mathrm{Sb})_{2} \mathrm{Te}_{3} / \mathrm{h}-\mathrm{BN}$ heterostructure acquired in (a) CTEM and (b) HAADF-STEM. The h-BN substrate is visible through holes in the $(\mathrm{Bi}, \mathrm{Sb})_{2} \mathrm{Te}_{3}$ film. Scale bars are $200 \mathrm{~nm}$. 